KEK-TH-679

\title{
Lepton Flavor Violation in the Randall-Sundrum Model with Bulk Neutrinos
}

\author{
Ryuichiro Kitano' \\ Theory Group, KEK, Oho 1-1, Tsukuba, Ibaraki 305-0801,Japan \\ and \\ Department of Particle and Nuclear Physics, The Graduate University for Advanced Studies, \\ Oho 1-1, Tsukuba, Ibaraki 305-0801, Japan
}

\begin{abstract}
Lepton flavor violation in the Randall-Sundrum model with bulk neutrinos is considered. Grossman and Neubert recently proposed that the existence of tiny neutrino masses and large mixing could be explained by the presence of the bulk neutrinos in the Randall-Sundrum background. Since the zero mode wave functions of the bulk neutrinos are localized on Planck brane, the Dirac Yukawa couplings on our brane are exponentially suppressed enough to generate tiny neutrino masses. However, the existence of KaluzaKlein modes of these bulk neutrinos enhance lepton flavor violating processes such as $\mu \rightarrow e \gamma$, from which lower bounds on their masses can be derived. We find that the first KK mode must be heavier than $25 \mathrm{TeV}$ if all the neutrino Yukawa couplings are of order unity, which requires a fine-tuning for the Higgs mass parameter.

PACS Numbers: 11.10.Kk, 13.35.Bv
\end{abstract}

\footnotetext{
${ }^{1}$ email: ryuichiro.kitano@kek.jp
} 
Recently, it was pointed out that the existence of extra-dimensions could be a solution of the hierarchy problem [1, 2]. In the Randall-Sundrum (RS) model, the electroweak scale is generated from the Planck scale parameters through the non-factorizable metric of the extra-dimension. In these model, however, it is not easy to explain small neutrino masses suggested by the neutrino oscillation data. In four dimensional theory, the seesaw mechanism is an elegant way of providing such tiny neutrino masses [3]. However, the RS scenario is incompatible with an intermediate scale such as the right-handed Majorana mass scale because all the dimensionful parameters on weak brane are suppressed by the exponential factor to electroweak scale. Recently a new mechanism for generating tiny neutrino masses was proposed by Grossman and Neubert [4. They introduce bulk fermion fields which couple to the lepton doublets with Dirac Yukawa couplings in the same way as right-handed neutrinos. In this scenario, the neutrino Dirac Yukawa couplings are exponentially suppressed in four dimensions because the zero mode wave functions of the bulk fermions are localized at the Planck brane and the tiny Dirac mass terms for neutrinos are generated.

The introduction of bulk fermions in the RS background leads to the existence of Kaluza-Klein $(\mathrm{KK})$ modes whose masses are at the electroweak scale in four dimensional theory, and the neutrino Dirac Yukawa couplings for these KK modes are not suppressed. Therefore sizable effects may arise in the phenomenology at the electroweak scale [5, 6, 7] because of the large mixing between KK modes and neutrinos. Grossman and Neubert pointed out that the invisible width of the $Z^{0}$ boson restricts this mixing.

In this paper, we consider the lepton flavor violating processes in the RS model with bulk neutrinos. The neutrino oscillation data implies that lepton flavor symmetry is violated. In the standard model with neutrinos, lepton flavor violation in the charged lepton sector is too small to be observed e.g. $\operatorname{Br}(\mu \rightarrow e \gamma) \sim 10^{-40}$ [8]. However with KK modes, the experimental bound for $\operatorname{Br}(\mu \rightarrow e \gamma)$ gives severe constraints on the mixing between the bulk fermions and neutrinos. If the Yukawa coupling is $O(1)$, the KK modes should be heavier than $25 \mathrm{TeV}$ which means the fine-tuning of the Higgs mass parameter is necessary to reproduce correct vacuum expectation values (VEVs).

The RS model is a five dimensional theory in which the fifth dimension is compactified to $S_{1} / \mathbf{Z}_{2}$ and two 3 -branes are located at two orbifold fixed points. The five dimensional metric is given as

$$
d s^{2}=e^{-2 k r_{c}|\phi|} \eta_{\mu \nu} d x^{\mu} d x^{\nu}-r_{c}^{2} d \phi^{2}
$$

where $k$ is a parameter of the order of the fundamental scale $M, r_{c}$ is the compactification radius which is also of the order of $M^{-1}$ and $\phi$ is the coordinate of the fifth dimension which is defined on $[0, \pi]$. The standard model fields are confined on the brane at $\phi=\pi$. In 
this set-up, the Planck scale $M_{\mathrm{Pl}}$ and the VEV for the Higgs field $v$ in the four dimensional effective theory are given as

$$
M_{\mathrm{Pl}}^{2}=\frac{M^{3}}{k}\left(1-e^{-2 k r_{c} \pi}\right), \quad v=e^{-k r_{c} \pi} v_{0},
$$

where $v_{0}$ is the VEV for the Higgs field in the five dimensional theory. We can see from eq.(2) that if we consider $M, k, 1 / r_{c}$ and $v_{0}$ as parameters of the order of $10^{19} \mathrm{GeV}$, all the dimensionful parameters on weak brane such as $v$ can be taken of the order of 100 $\mathrm{GeV}$ for $k r_{c} \sim 12$ while keeping $M_{\mathrm{Pl}}$ close to $10^{19} \mathrm{GeV}$.

The action of the bulk fermion in the RS set-up is given by [4]

$$
S=\int d^{4} x \int_{0}^{\pi} d \phi \sqrt{G}\left\{\frac{i}{2} \bar{\Psi} \hat{\gamma}^{A} \partial_{A} \Psi-\frac{i}{2}\left(\partial_{A} \bar{\Psi}\right) \hat{\gamma}^{A} \Psi-m \operatorname{sgn}(\phi) \bar{\Psi} \Psi\right\} .
$$

The Majorana mass term is omitted by imposing lepton number conservation. KaluzaKlein decomposition of $\Psi$ reduces this action to the usual four dimensional Dirac action which is given by

$$
S=\sum_{n} \int d^{4} x\left\{\bar{\psi}_{n}(x) i \not \partial \psi_{n}(x)-m_{n} \bar{\psi}_{n}(x) \psi_{n}(x)\right\}
$$

where $\psi \equiv \psi^{L}+\psi^{R}$, and $\psi^{L, R}$ is defined as

$$
\Psi_{L, R}(x, \phi) \equiv \frac{1}{2}\left(1 \mp \gamma_{5}\right) \Psi=\frac{e^{2 k r_{c}|\phi|}}{\sqrt{r_{c}}} \sum_{n} \psi_{n}^{L, R}(x) \hat{f}_{n}^{L, R}(\phi) .
$$

The wave functions $\hat{f}_{n}^{L, R}$ and the masses of KK modes $m_{n}$ are expressed by the following parameters:

$$
\epsilon=e^{-k r_{c} \pi}, t=\epsilon e^{k r_{c}|\phi|}, \nu=\frac{m}{k},
$$

where the parameter $\epsilon$ should be fixed as $\epsilon \sim 10^{-16}$ i.e. $k r_{c} \sim 12$ in order to produce the hierarchy between the Planck scale and the electroweak scale. The parameter $t \in[\epsilon, 1]$ is the redefined spatial coordinate of the fifth dimension; $t=\epsilon$ is the location of the Planck brane and $t=1$ is that of our brane. By rescaling the function as $\hat{f}_{n}^{L, R}(\phi) \rightarrow \sqrt{k r_{c} \epsilon} f_{n}^{L, R}(t)$, the wave functions and the masses are explicitly given as

$$
\begin{aligned}
& f_{0}^{L}(t)=0, f_{0}^{R}(t)=\sqrt{\frac{1-2 \nu}{1-\epsilon^{1-2 \nu}}} t^{-\nu}, \\
& f_{n}^{L, R}(t)=\frac{\sqrt{2 t}}{J_{\nu+\frac{1}{2}}\left(x_{n}\right)} J_{\nu \mp \frac{1}{2}}\left(x_{n} t\right) \quad\left(n \neq 0, \nu>\frac{1}{2}\right),
\end{aligned}
$$




$$
m_{n}=\epsilon k x_{n}
$$

where $x_{n}$ is the solution of $J_{\nu-\frac{1}{2}}\left(x_{n}\right)=0$. For $\epsilon \sim 10^{-16}$, the masses of the KK modes $m_{n}$ are of the order of the electroweak scale. The zero mode wave function (there is a zero mode $f_{0}^{R}$ since $x_{0}=0$.) on our brane is very suppressed such as $f_{0}^{R}(1) \propto \epsilon^{\nu-\frac{1}{2}}$ for $\nu>1 / 2$. This smallness is the origin of the tiny neutrino masses. The wave functions of KK modes are not suppressed $\left(f_{n}^{R}(1)=\sqrt{2}\right)$ and this gives large coupling constants which violate lepton flavor symmetry.

In this theory, even numbers of bulk fermions are needed to cancel the parity anomaly of the $\mathbf{Z}_{2}$ orbifold symmetry [9, 10]. For simplicity, we consider the minimal case in which there are two bulk fermions $\Psi^{\alpha}(\alpha=1,2)$.

We can construct the gauge invariant interaction terms between the lepton doublets $L_{0}^{i}=\left(\nu_{0 L}^{i}, e_{0 L}^{i}\right)(i=e, \mu, \tau)$ and the bulk fermions $\Psi^{\alpha}(\alpha=1,2)$ as

$$
S_{Y}=-\int d^{4} x e^{-4 k r_{c} \pi}\left\{\hat{y}_{i \alpha} \bar{L}_{0}^{i}(x) \widetilde{H}_{0}(x) \Psi_{R}^{\alpha}(x, \pi)+\text { h.c. }\right\}
$$

where $\widetilde{H_{0}} \equiv i \sigma_{2} H_{0}^{*}=\left(H_{0}^{0 *}, H_{0}^{-}\right)$is Higgs field. The couplings $\hat{y}_{i \alpha}$ are dimensionful parameters which are naturally of the order of $M_{\mathrm{Pl}}^{-1 / 2}$. In four dimensional effective theory, this action is written in terms of $\psi$ as

$$
S_{Y}=-\sum_{n \geq 0} \int d^{4} x\left\{y_{n}^{i \alpha} \bar{L}^{i}(x) \widetilde{H}(x) \psi_{n, \alpha}^{R}(x)+\text { h.c. }\right\},
$$

where the lepton doublets $L$ and the Higgs doublet $\widetilde{H} \equiv i \sigma_{2} H^{*}$ are properly rescaled to give canonical kinetic terms in four dimensions. The relation of $\hat{y}_{i \alpha}$ and $y_{n}^{i \alpha}$ is

$$
y_{n}^{i \alpha}=\sqrt{k} \hat{y}_{i \alpha} f_{n}^{R}(1) \equiv z_{i \alpha} f_{n}^{R}(1) .
$$

Here we take that $z_{i \alpha}$ to be parameters of order unity. From eq.(1) and eq.(11), the mass matrix for neutrinos is given by

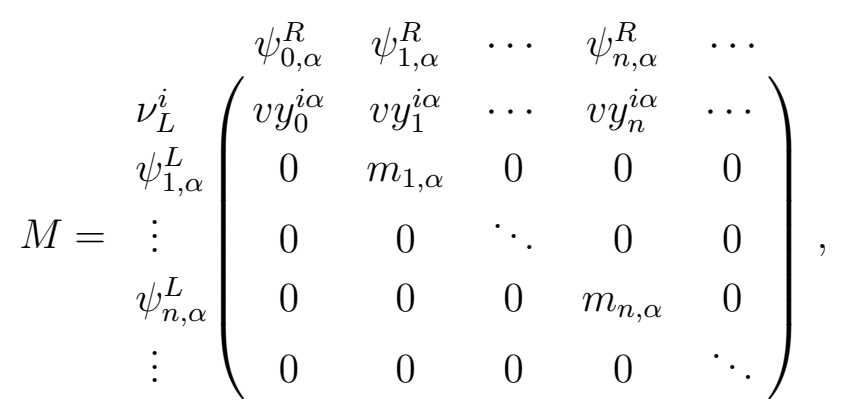

where $m_{n, \alpha}$ (from eq.(4)) is the mass of the $n$-th KK mode for $\Psi^{\alpha}$. For appropriate choices of $\nu(\nu \sim 1), y_{0}^{i \alpha}\left(\equiv z_{i \alpha} f_{0}^{R}(1)\right)$ becomes small enough to explain the tiny neutrino 
masses. In four dimensional effective theory, this model also contains a series of vector-like neutrinos which may lead to sizable lepton flavor violation [11].

The $3 \times 2$ submatrix for the light neutrinos is written as

$$
M_{\mathrm{sub}}=\left(\begin{array}{cc}
\sqrt{2 \nu_{1}-1} \epsilon^{\nu_{1}-\frac{1}{2}} z_{e 1} v & \sqrt{2 \nu_{2}-1} \epsilon^{\nu_{2}-\frac{1}{2}} z_{e 2} v \\
\sqrt{2 \nu_{1}-1} \epsilon^{\nu_{1}-\frac{1}{2}} z_{\mu 1} v & \sqrt{2 \nu_{2}-1} \epsilon^{\nu_{2}-\frac{1}{2}} z_{\mu 2} v \\
\sqrt{2 \nu_{1}-1} \epsilon^{\nu_{1}-\frac{1}{2}} z_{\tau 1} v & \sqrt{2 \nu_{2}-1} \epsilon^{\nu_{2}-\frac{1}{2}} z_{\tau 2} v
\end{array}\right),
$$

where $\nu_{\alpha}=m_{\alpha} / k(\alpha=1,2)$ and $m_{\alpha}$ is the mass of the bulk fermions (see eq.(3)). We take here $m_{1}>m_{2}$. Since this matrix is $3 \times 2$, one of the three light neutrinos remains massless.

Now we discuss the lepton flavor violating processes such as $\mu \rightarrow e \gamma, \tau \rightarrow \mu \gamma$ and $\tau \rightarrow e \gamma$. The experimental bounds for these processes give severe constraints on the mass of the KK modes and/or couplings $z_{i \alpha}$.

The four dimensional gauge and Yukawa interaction terms relevant to $\mu \rightarrow e \gamma, \tau \rightarrow \mu \gamma$ and $\tau \rightarrow e \gamma$ process are given by

$$
\begin{aligned}
\mathcal{L}^{\text {gauge }}= & \sum_{i=e, \mu, \tau} \frac{g}{\sqrt{2}} W_{\mu}^{\dagger} \bar{e}_{L}^{i} \gamma^{\mu} P_{L} \nu_{L}^{i}+\text { h.c. } \\
= & \sum_{i=e, \mu, \tau} \sum_{A=1}^{2 N+3} \frac{g}{\sqrt{2}} U_{i A} W_{\mu}^{\dagger} \bar{e}_{L}^{i} \gamma^{\mu} P_{L} \psi_{\nu}^{A}+\text { h.c. } \\
\mathcal{L}^{\text {Yukawa }}= & \sum_{n=0}^{N} \sum_{i=e, \mu, \tau} \sum_{\alpha=1,2} y_{n}^{i \alpha} \bar{e}_{L}^{i} H^{-} P_{R} \psi_{n, \alpha}^{R}-\sum_{i=e, \mu, \tau} f^{i} \bar{\nu}_{L}^{i} H^{+} P_{R} e_{R}^{i}+\text { h.c. } \\
= & \sum_{n=0}^{N} \sum_{i=e, \mu, \tau} \sum_{\alpha=1,2} \sum_{A=1}^{N} y_{n}^{i \alpha} V_{(n, \alpha) A} \bar{e}_{L}^{i} H^{-} P_{R} \psi_{\nu}^{A} \\
& -\sum_{i=e, \mu, \tau} \sum_{A=1}^{N} f^{i} U_{i A}^{*} \bar{\psi}_{\nu}^{A} H^{+} P_{R} e_{R}^{i}+\text { h.c. },
\end{aligned}
$$

where the indices $i, A, n, \alpha$ represent the flavor, the mass eigenstates of neutrinos, the KK excitations and the species of bulk fermions, respectively. Here the left-handed mixing matrix $U$ and the right-handed mixing matrix $V$ are defined as the matrices which diagonalize $M M^{\dagger}$ and $M^{\dagger} M$, respectively. To cut-off the infinite KK modes we introduce $N$ and consider up to $N$-th KK mode. Then $U$ and $V$ are $(2 N+3) \times(2 N+3)$, $(2 N+2) \times(2 N+2)$ matrices respectively. The coupling $f^{i}$ is the lepton Dirac Yukawa coupling of the $i$-th generation and $y_{n}^{i \alpha}$ are neutrino Dirac Yukawa couplings defined in eq.(11). The field $\psi_{\nu}^{A}$ represents the $A$-th mass eigenstate of the neutrinos. 
We first calculate $\operatorname{Br}(\mu \rightarrow e \gamma) \cdot \operatorname{Br}(\tau \rightarrow \mu \gamma)$ and $\operatorname{Br}(\tau \rightarrow e \gamma)$ can be calculated in the same way. The decay amplitude of $\mu \rightarrow e \gamma$ is generally given by

$$
T(\mu \rightarrow e \gamma)=e \epsilon^{\alpha *} \bar{u}_{e}(p-q)\left[i \sigma_{\alpha \beta} q^{\beta}\left(A_{L} P_{L}+A_{R} P_{R}\right)\right] u_{\mu}(p)
$$

where $P_{L}$ and $P_{R}$ are the chiral projection operators. The decay width is given by

$$
\Gamma(\mu \rightarrow e \gamma)=\frac{e^{2}}{16 \pi} m_{\mu}^{3}\left(\left|A_{L}\right|^{2}+\left|A_{R}\right|^{2}\right),
$$

where $m_{\mu}$ is the mass of the muon. Neglecting the mass of the electron, $A_{L}$ and $A_{R}$ can be expressed as

$$
\begin{aligned}
& A_{L}=0 \\
& \begin{aligned}
A_{R}= & \frac{g^{2}}{(4 \pi)^{2}} \sum_{A} \frac{m_{\mu}}{M_{W}^{2}} U_{e A} U_{\mu A}^{*} \\
& \quad \times \frac{1}{24\left(1-\xi_{A}\right)}\left(10-43 \xi_{A}+78 \xi_{A}^{2}-49 \xi_{A}^{3}+4 \xi_{A}^{4}+18 \xi_{A}^{3} \log \xi_{A}\right), \\
& \left(\xi_{A} \equiv m_{A}^{2} / M_{W}^{2}\right)
\end{aligned}
\end{aligned}
$$

where $M_{W}$ is $W$ boson mass and $m_{A}$ is the mass of the $A$-th mass eigenstates of neutrinos. Notice that this model predicts $\mu^{-} \rightarrow e_{L}^{-} \gamma\left(\right.$ or $\left.\mu^{+} \rightarrow e_{R}^{+} \gamma\right)$ decay. If all the neutrino masses are small, this amplitude is suppressed by the GIM mechanism [12]. However, due to the existence of heavy neutrinos, the GIM cancellation does not work and $A_{R}$ is estimated approximately as

$$
A_{R} \simeq \frac{m_{\mu}}{(4 \pi)^{2}} \sum_{n=1}^{N}\left(\frac{z_{e 1} z_{\mu 1}}{m_{n, 1}^{2}}+\frac{z_{e 2} z_{\mu 2}}{m_{n, 2}^{2}}\right) .
$$

and from eq.(9), this is written as

$$
\begin{aligned}
& A_{R} \simeq \frac{1}{(4 \pi)^{2}} \frac{m_{\mu}}{(\epsilon k)^{2}}\left(z_{e 1} z_{\mu 1} C_{1}(N)+z_{e 2} z_{\mu 2} C_{2}(N)\right), \\
& C_{\alpha}(N)=\sum_{n=1}^{N} \frac{1}{x_{n, \alpha}^{2}},(\alpha=1,2),
\end{aligned}
$$

where $x_{n, \alpha}$ are the solutions for $J_{\nu_{\alpha}-\frac{1}{2}}\left(x_{n, \alpha}\right)=0$. The functions $C_{\alpha}(N)$ are slowly increasing functions of $N$, and therefore the cut off dependence of $\operatorname{Br}(\mu \rightarrow e \gamma)$ is small. In the limit of $N \rightarrow \infty, C_{\alpha}(N) \rightarrow\left(2\left(2 \nu_{\alpha}+1\right)\right)^{-1}$.

The branching ratio is given by eq.(22) as

$$
\operatorname{Br}(\mu \rightarrow e \gamma) \simeq 0.0037\left(\frac{v}{\epsilon k}\right)^{4}\left|z_{e 1} z_{\mu 1} C_{1}(N)+z_{e 2} z_{\mu 2} C_{2}(N)\right|^{2}
$$


The same calculation for $\tau \rightarrow \mu \gamma$ and $\tau \rightarrow e \gamma$ gives

$$
\begin{aligned}
& \operatorname{Br}(\tau \rightarrow \mu \gamma) \simeq 0.00065\left(\frac{v}{\epsilon k}\right)^{4}\left|z_{\mu 1} z_{\tau 1} C_{1}(N)+z_{\mu 2} z_{\tau 2} C_{2}(N)\right|^{2} \\
& \operatorname{Br}(\tau \rightarrow e \gamma) \simeq 0.00065\left(\frac{v}{\epsilon k}\right)^{4}\left|z_{e 1} z_{\tau 1} C_{1}(N)+z_{e 2} z_{\tau 2} C_{2}(N)\right|^{2} .
\end{aligned}
$$

The present experimental bounds are $\operatorname{Br}(\mu \rightarrow e \gamma)<1.2 \times 10^{-11}$ 《13, $\operatorname{Br}(\tau \rightarrow \mu \gamma)<$ $1.1 \times 10^{-6}$ 14 and $\operatorname{Br}(\tau \rightarrow e \gamma)<2.7 \times 10^{-6}$ [15. If all $z_{i \alpha}$ are of order unity, the dimensionless combination $v / \epsilon k$ must satisfy

$$
\frac{v}{\epsilon k} \lesssim 0.02
$$

from the constraint from $\operatorname{Br}(\mu \rightarrow e \gamma)$. In eq. (27), we use $C_{\alpha}(N) \sim\left(2\left(2 \nu_{\alpha}+1\right)\right)^{-1}$ and $\nu_{\alpha} \sim 1$ which is a reasonable region for producing light neutrino masses. The parameter $x_{1, \alpha}$ is roughly estimated to be $x_{1, \alpha} \sim 3$ for $\nu_{\alpha} \sim 1$, so that we can derive the following bound for the lowest KK mode $m_{\mathrm{KK}}$ from eq.(9) and eq.(27):

$$
m_{\mathrm{KK}} \gtrsim 25 \mathrm{TeV}
$$

Since this value is two order of magnitude larger than the Higgs VEV, a fine-tuning of $10^{-2}$ is necessary.

An individual constraint on $z_{i \alpha}$ can be obtained by considering the neutrino oscillation data. To reproduce the mixing angle $\sin ^{2} 2 \theta_{12} \sim 10^{-2}$ for the small angle MSW solution, $\sin ^{2} 2 \theta_{12} \sim 1$ for the large angle MSW solution, $\sin ^{2} 2 \theta_{23} \sim 1$ to explain the atmospheric neutrino anomaly and $\sin ^{2} 2 \theta_{13} \lesssim 0.1$ from the CHOOZ experiment [16], the structure of the Yukawa couplings are roughly given by

$$
\begin{aligned}
& \left|z_{e 1}\right| \sim x\left|z_{\mu 1}\right| \sim x\left|z_{\tau 1}\right|, \\
& \left|z_{e 2}\right| \ll\left|z_{\mu 2}\right| \sim\left|z_{\tau 2}\right|
\end{aligned}
$$

where $x \sim 14,1 / 28$ for the small angle MSW solution and $x \sim 0.7$ for the large angle MSW solution [4]. Therefore the restrictions on $z_{i 1}$ are given as

$$
\begin{aligned}
& \frac{v\left|z_{e 1}\right|}{\epsilon k} \lesssim 0.08, \frac{v\left|z_{\mu 1}\right|}{\epsilon k} \sim \frac{v\left|z_{\tau 1}\right|}{\epsilon k} \lesssim 0.006(x=14) \\
& \frac{v\left|z_{e 1}\right|}{\epsilon k} \lesssim 0.004, \frac{v\left|z_{\mu 1}\right|}{\epsilon k} \sim \frac{v\left|z_{\tau 1}\right|}{\epsilon k} \lesssim 0.1\left(x=\frac{1}{28}\right) \\
& \frac{v\left|z_{e 1}\right|}{\epsilon k} \sim \frac{v\left|z_{\mu 1}\right|}{\epsilon k} \sim \frac{v\left|z_{\tau 1}\right|}{\epsilon k} \lesssim 0.02(x=0.7) .
\end{aligned}
$$


Since we only know the upper bound on the mixing angle $\sin ^{2} 2 \theta_{13}$, typically we take $\sin ^{2} 2 \theta_{13}=0.05$. Then the constraints on $z_{i 2}$ are given by

$$
\frac{v\left|z_{e 2}\right|}{\epsilon k} \lesssim 0.009, \frac{v\left|z_{\mu 2}\right|}{\epsilon k} \sim \frac{v\left|z_{\tau 2}\right|}{\epsilon k} \lesssim 0.05 .
$$

In ref. [四, the constraint $v\left|z_{i \alpha}\right| / \epsilon k \lesssim 0.1$ was derived from the invisible decay width of the $Z^{0}$ boson i.e. the deviation from unitarity of the MNS matrix which is $3 \times 3$ submatrix of the matrix $U$ [17]. We can find more severe constraints from considering lepton flavor violation. The smallness of $v\left|z_{i \alpha}\right| / \epsilon k$ means that the five dimensional Yukawa couplings

$\hat{y}_{i \alpha}$ or the five dimensional VEV of the Higgs field $v_{0}$ should be much smaller than $M_{\mathrm{Pl}}^{-1 / 2}$ or $M_{\mathrm{Pl}}$, respectively, which is the only natural scale of the original parameters. In this sense, the bounds in eq.(31 34) are considered to be somewhat unnatural.

In conclusion, we have considered lepton flavor violating processes in the context of the small extra-dimension scenario. The neutrino mass and mixing needs right-handed neutrinos, but naive introduction of the right-handed neutrino does not provide tiny neutrino masses. Grossman and Neubert then proposed the existence of right-handed neutrinos which live in bulk and couple to the lepton doublets and we saw that this model lead to small Dirac neutrino mass terms. The neutrino mass and mixing causes lepton flavor violation. The KK modes of the right-handed neutrinos enhance the branching ratio of these processes. We calculated the $\operatorname{Br}(\mu \rightarrow e \gamma), \operatorname{Br}(\tau \rightarrow \mu \gamma)$ and $\operatorname{Br}(\tau \rightarrow e \gamma)$ and found that $\operatorname{Br}(\mu \rightarrow e \gamma)$ gives severe constraints on the neutrino Yukawa couplings $\hat{y}_{i \alpha}$ and/or the Higgs mass parameter $v_{0}$ in the five dimensional theory.

\section{Acknowledgments}

The author would like to thank Y. Okada, J. Hisano and A. Akeroyd for reading manuscript and useful comments. He also thanks K. Okumura and S. Kiyoura for discussions.

\section{References}

[1] N. Arkani-Hamed, S. Dimopoulos and G. Dvali, Phys. Lett. B429, 263 (1998) hep$\mathrm{ph} / 9803315$.

I. Antoniadis, N. Arkani-Hamed, S. Dimopoulos and G. Dvali, Phys. Lett. B436, 257 (1998) hep-ph/9804398.

[2] L. Randall and R. Sundrum, Phys. Rev. Lett. 83, 3370 (1999) hep-ph/9905221].

[3] T. Yanagida, in Proceedings of the Workshop on Unified Theory and Baryon Number of the Universe, eds. O. Sawada and A. Sugamoto (KEK, 1979) p.95; 
M. Gell-Mann, P. Ramond, and R. Slanski, in Supergravity, eds. P. van Nieuwenhuizen and D. Freedman (North Holland, Amsterdam, 1979).

[4] Y. Grossman and M. Neubert, hep-ph/9912408.

[5] S. Chang, J. Hisano, H. Nakano, N. Okada and M. Yamaguchi, hep-ph/9912498.

[6] H. Davoudiasl, J. L. Hewett and T. G. Rizzo, hep-ph/9911262.

[7] A. Pomarol, hep-ph/9911294.

[8] S. T. Petcov, Sov. J. Nucl. Phys. 25, 340 (1977).

[9] A. N. Redlich, Phys. Rev. Lett. 52, 18 (1984).

[10] C. G. Callan and J. A. Harvey, Nucl. Phys. B250, 427 (1985).

[11] T. Cheng and L. Li, Phys. Rev. D16, 1425 (1977). T. P. Cheng and L. Li, Phys. Rev. Lett. 38, 381 (1977). J. D. Bjorken, K. Lane and S. Weinberg, Phys. Rev. D16, 1474 (1977). B. W. Lee, S. Pakvasa, R. E. Shrock and H. Sugawara, Phys. Rev. Lett. 38, 937 (1977). B. W. Lee and R. E. Shrock, Phys. Rev. D16, 1444 (1977). G. Altarelli, L. Baulieu, N. Cabibbo, L. Maiani and R. Petronzio, Nucl. Phys. B125, 285 (1977). P. Langacker and D. London, Phys. Rev. D38, 886 (1988). P. Langacker and D. London, Phys. Rev. D38, 907 (1988). A. Ilakovac, Nucl. Phys. Proc. Suppl. 76, 193 (1999).

[12] S. L. Glashow, J. Iliopoulos and L. Maiani, Phys. Rev. D2, 1285 (1970).

[13] M. L. Brooks et al. [MEGA Collaboration], Phys. Rev. Lett. 83, 1521 (1999) hepex/9905013].

[14] S. Ahmed et al. [CLEO Collaboration], hep-ex/9910060.

[15] K. W. Edwards et al. [CLEO Collaboration], Phys. Rev. D55, 3919 (1997).

[16] Y. Fukuda et al. [Super-Kamiokande Collaboration], Phys. Rev. Lett. 81, 1562 (1998) hep-ex/9807003.

M. Nakahata [Super-Kamiokande Collaboration], Nucl. Phys. Proc. Suppl. 76, 425 (1999).

M. Apollonio et al., Phys. Lett. B466, 415 (1999) hep-ex/9907037.

[17] Z. Maki, M. Nakagawa and S. Sakata, Prog. Theor. Phys. 28, 870 (1962). 